\title{
Development of Retinal Vasculature Is Mediated by Hypoxia- Induced Vascular Endothelial Growth Factor (VEGF) Expression by Neuroglia
}

\author{
Jonathan Stone, ${ }^{1}$ Ahuva Itin, ${ }^{2}$ Tamar Alon, ${ }^{2}$ Jacob Pe'er, ${ }^{3}$ Hadassah Gnessin, ${ }^{3}$ Tailoi Chan-Ling, ${ }^{1}$ and Eli \\ Keshet $^{2}$ \\ 'Department of Anatomy and Histology, University of Sydney, Australia and Departments of ${ }^{2}$ Molecular Biology and \\ ${ }^{3}$ Ophthalmology, Hebrew University and Hadassah University Hospital, Jerusalem 91120, Israel
}

We have studied the role of the hypoxia-inducible angiogenic growth factor vascular endothelial growth factor (VEGF) in the induction and control of vessel growth in the developing retina of rats and cats, using in situ hybridization techniques. VEGF is expressed successively in two layers of neural retina, the innermost (axon) layer and the inner nuclear layer (INL). In the axon layer, VEGF is expressed transiently by astrocytes as they spread across the layer, closely preceding the formation of superficial vessels. In the INL, VEGF is expressed transiently by somas at the middle of the layer (presumably Müller cells), closely preceding the formation of the deep layer of retinal vessels.

We propose that hypoxia caused by the onset of neuronal activity is detected by strategically located populations of neuroglia, first astrocytes, then Müller cells. In response they secrete VEGF, inducing formation of the superficial and deep layers of retinal vessels, respectively. As the vessels become patent, they relieve the hypoxic stimulus, so vessel formation is matched to oxygen demand. This hypothesis was tested experimentally in three ways. Expression of the high affinity $f / k-1$ receptor for VEGF was demonstrated in newly formed retinal vessels, confirming that the secreted VEGF acts on the vessels, in a paracrine fashion. Direct hypoxic regulation of VEGF expression by macroglia was demonstrated in primary cultures of astrocytes and in cells of a glioma line. Hypoxic regulation of VEGF expression in the intact developing retina was demonstrated by showing that oxygen-enriched atmospheres that inhibit vessel formation also suppress endogenous VEGF production.

[Key words: vascular endothelial growth factor (VEGF), hypoxia, astrocytes, Müller cells, angiogenesis, retina]

The development of the retinal vasculature is considered to be regulated by oxygen levels in retinal tissue, and mediated by a vasoformative function produced by the retina itself. In this study we have used in situ hybridization techniques to test the roles of the macroglia of the retina, and a hypoxia-inducible

\footnotetext{
Received Nov. 22, 1994; revised Feb. 1, 1995; accepted Feb. 3, 1995.

The work was supported by grants from the Basic Research Foundation of the Israeli Academy of Sciences, and the National Health and Medical Research Council of Australia.

Correspondence should be addressed to Dr. Eli Keshet at the above address. Copyright (c) 1995 Society for Neuroscience 0270-6474/95/154738-10\$05.00/0
}

angiogenic growth factor in linking tissue oxygen to vessel growth. The importance of oxygen in the control of vessel development in the retina was demonstrated by Ashton (1966, 1968), as he explored the role of oxygen in the pathogenesis of a disease of the developing vasculature, retinopathy of prematurity. Subsequent studies confirmed the importance of oxygen which, in the retina, can be manipulated by changing the oxygen content of inspired air. Phelps (1990), for example, showed that oxygen controls the microarchitecture of retinal vessels, and Chan-Ling et al. (1990) showed that the pattern of vessel growth matches the pattern of maturation of retinal neurones. Reviewing this evidence, Chan-Ling and Stone (1993) proposed that physiological levels of hypoxia are the stimulus for normal development of both the superficial and deep layer of the retinal vasculature. The action of a retina-derived growth factor in controlling the formation of retinal vessels was first proposed by Michaelson (1954) and confirmed in several subsequent studies (Glaser et al., 1980, D'Amore et al., 1981; Chen and Chen, 1980, 1984, 1987).

Vascular endothelial growth factor (VEGF) is a secreted, 46 $\mathrm{kDa}$ dimeric glycoprotein and a potent angiogenic factor, with actions as an endothelial cell-specific mitogen and chemoattractant, and as a vascular permeability factor (Gospodarowitz et al., 1989; Connolly, 1991; Ferrara et al., 1991). Anti-VEGF antibodies and a reagent interfering with VEGF/VEGF receptor interaction block neovascularization of VEGF-producing tumors (Kim et al., 1993; Millauer et al., 1994). In the embryo, and during the ovarian cycle, VEGF is expressed in spatial and temporal patterns consistent with a role as a paracrine angiogenic factor (Breier et al., 1992; Jakeman et al., 1993; Shweiki et al., 1993). We have recently shown that VEGF expression is induced by hypoxia (Shweiki et al., 1992), so that VEGF is an attractive candidate for a vasoformative growth factor of the retina.

We therefore examined the expression of VEGF in the developing retina of the rat and the cat, in which the retina becomes vascularized in late fetal and early neonatal life, in a pattern characteristic of many mammals, including the human (Michaelson, 1954). Our results confirm the role of VEGF in the development of retinal vessels and show the importance of the macroglia in the process. We hypothesize that the macroglial cells of the retina serve to detect the physiological levels of hypoxia which induce normal vessel formation and respond by secreting VEGF. The secreted VEGF causes the formation of the retinal vessels, whose function reduces the hypoxic stimulus for 
vessel formation, thus matching oxygen supply to demand. This hypothesis was tested and confirmed by showing that developing retinal vessels express VEGF receptors, that both primary cultures of astrocytes and glioma cell lines upregulate VEGF in response to hypoxia, and that hyperoxia in vivo, which inhibits vessel formation, downregulates VEGF expression.

\section{Materials and Methods}

Material studied. Rctinas studied were from common cats aged from $\mathbf{P}$ (postnatal day) 4 to 15, and from Sabra albino rats aged from P0 to P14. Animals were given an overdose $(60 \mathrm{mg} / \mathrm{kg})$ of sodium pentobarbitone and the cats were perfused transcardially with phosphate-buffered saline (PBS) at $\mathrm{pH} 7.4$ followed by $4 \%$ paraformaldehyde in PBS. The eyes were enucleated immediately post mortem and immersed in $4 \%$ paraformaldehyde in PBS. Fixation times ranged from $1 \mathrm{~d}$ to several weeks, without affecting the techniques applied.

Exposure to hyperoxia. The effect of hyperoxia on VEGF expression was examined in retinas from rats aged P 3 and P10. Half the animals were raised in $70-80 \%$ oxygen for $2-3 \mathrm{~d}$, with their mothers; the remainder were littermate controls raised in normoxia. The oxygen-enriched atmosphere was obtained by mixing air and oxygen. Oxygen levels were monitored by a polargraphic oxygen sensor (Commonwealth Industrial Gases, Australia).

Lectin histochemistry and GFAP immunocytochemistry. Rat retinas were dissected in PBS and prepared as whole mounts. The sclera and choroid were carefully cleaned from the outer surface of the retina, and hyaloid vessels and vitreous humor from the inner surface. Retinas were prepared for immunohistochemistry as previously described (Chan-Ling and Stone, 1991). Briefly, radial incisions were made to permit flattening of the retina, which was then fixed by immersion in $0.5 \%$ paraformaldehyde, postfixed in cold $70 \%$ ethanol, and washed in PBS containing $1 \%$ Triton X-100.

To show the relationship between retinal vasculature and astrocytes, blood vessels were visualized with the Bandeira simplicifolia isolectin B4, (FITC-conjugated, Sigma) and astrocytes were visualized with a polyclonal anti-glial fibrillary acidic protein (GFAP) antibody (Sigma) and a TRITC conjugated goat anti-rabbit secondary antibody (Sigma), using protocols described previously (Chan-Ling and Stone, 1992).

Combined lectin and bromodeoxyuridine immunohistochemistry. Proliferating endothelial cells were identified by their incorporation of the thymidine analogue 5 '-bromodeoxyuridine $(0.1 \mathrm{mg} / \mathrm{kg}$, given intraperitoneally) and visualized in retinal whole mounts. The retinas were first incubated with the Bandeira simplicifolia isolectin B4 and then with a monoclonal antibody to BrdU (kindly made available by M. Raff, University College London).

In situ hybridization. Paraffin-embedded eyes were sectioned (5-10 $\mu \mathrm{m}$ thick), processed and hybridized in situ as previously described (Shweiki et al., 1992). Autoradiographic exposure was for 5-9 d. As a VEGF-specific probe we used a $1.8 \mathrm{~kb}$ long cDNA fragment containing approximately the $3^{\prime}$ two-thirds of the coding region, as well as the entire $3^{\prime}$-untranslated region of mouse $V_{E G F}$. $\Lambda \mathrm{s}$ a VEGF receptorspecific probe of the KDR/Flk-1 type (Millauer et al., 1993) we used KDR cDNA (Terman et al., 1991). cDNAs were subcloned onto the polylinker of a PBS vector (Stratagente) and were linearized by digestion with the appropriate restriction endonuclease to allow synthesis of a ${ }^{35}$ S-labeled complementary RNA in either the antisense or sense orientation (using T3 or 'I"/ RNA polymerase, respectively). RNA probes were fragmented by mild alkaline treatment prior to use for in situ hybridization.

Cell culture and exposure to hypoxia. Primary astrocytes were isolated from neonatal rat brain as described by Frangakis and Kimelberg (1984). C6 cells, a clonal glial cell line derived from a rat glial tumor, were grown in Dulbecco-modified Eagle's medium (DMEM) supplemented with $5 \%$ fetal calf serum (FCS) and antibiotics. To achieve hypoxia, near-confluent cultures were incubated in a GasPak Plus an aerobic culture chamber (BBL Microbiology Systems), utilizing hydrogen and a palladium catalyst to remove all traces of oxygen. Exposure to hypoxic conditions was for $18 \mathrm{hr}$.

Reverse transcriptase-PCR analysis. Complementary DNA was synthesized from $300 \mathrm{ng}$ of total RNA using oligo(dT) as a primer and AMV reverse transcriptase. PCR amplification was carried out in the presence of a ${ }^{32} \mathrm{P}-\mathrm{dCTP}$ tracer $(2 \mu \mathrm{Ci}$ in a $100 \mu \mathrm{l}$ reaction volume), 1 mM of each dNTP, $2.5 \mathrm{mM} \mathrm{MgCl}_{2}$, and $2.5 \mathrm{U}$ of Taq polymerase. Twenty-five amplification cycles were used, consisting each of a $1 \mathrm{~min}$ in-

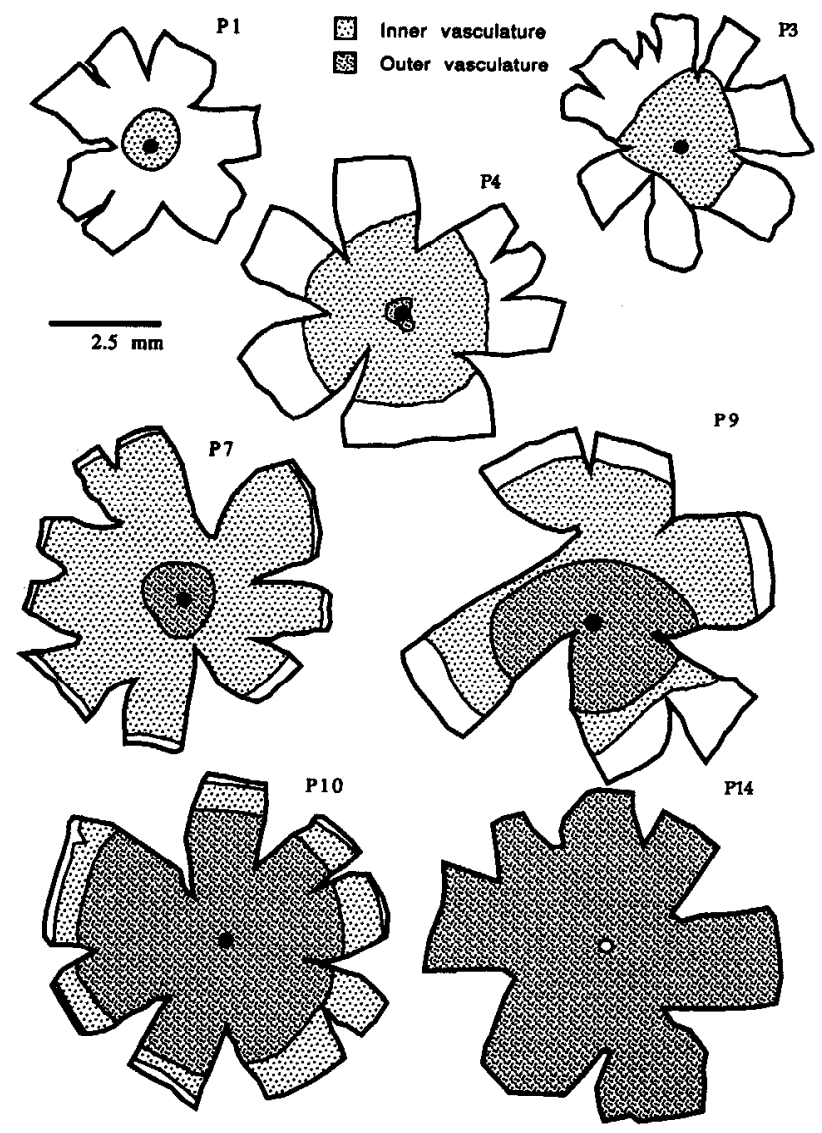

Figure 1. Maps of the development of retinal vasculature of the Sabra rat. Each diagram shows the outline of a whole-mounted retina, in which radial cuts have been made to allow the retina to be flattened. The optic disk is indicated as a small solid circle. The light shading shows the extent of spread of the superficial layer of vasculature, and the dark shading shows the extent of spread of the deep layer. The area shown for the deep vasculature includes areas where the deeper vessels have begun to bud from the superficial, even though the outer plexus of vessels is not fully formed.

cubation at $94^{\circ} \mathrm{C}$, a 2 min incubation at $65^{\circ} \mathrm{C}$, and a 3 min incubation at $72^{\circ} \mathrm{C}$. cDNAs were coamplified with a pair of VEGF-specific oligonucleotides and a pair of L19 ribosomal protein-specific oligonucleotides (serving as an internal standard). The following oligonucleotides were used. (1) GGAGAGATGAGCTTCCTACAG and TCACCGCCTTGGCTTGTCACA, corresponding to amino-acids $92-98$ and to the six carboxy-terminal amino-acids of VEGF, respectively. (2) CTGAAGGTCAAAGGGAATGTG and GGACAGAGTCTTGATGATCTC, corresponding to forward and reverse sequences, respectively, of L19. Amplified fragments were resolved in $6 \%$ nondenaturing polyacrylamide gel and were visualized by autoradiography.

\section{Results}

Timing and topography of retinal vascularization. Aspects of vessel formation in the retina of the Sabra rat are summarized in Figure 1. The superficial layer of vessels forms first, extending across the surface of the retina, from the optic disk. The process begins in late gestation and, in the youngest retina examined (P1), vessels had formed over the central $9 \%$ of the retina. Thereafter, the supcrficial vcssels spread steadily, reaching the edge of the retina by P12. The first-formed vessels are capillaries which are subsequently remodeled to their mature form, in particular by the formation of arteries and veins.

In a second process, vessels arise as buds from the superficial layer and grow radially outward (towards the sclera), as far as 

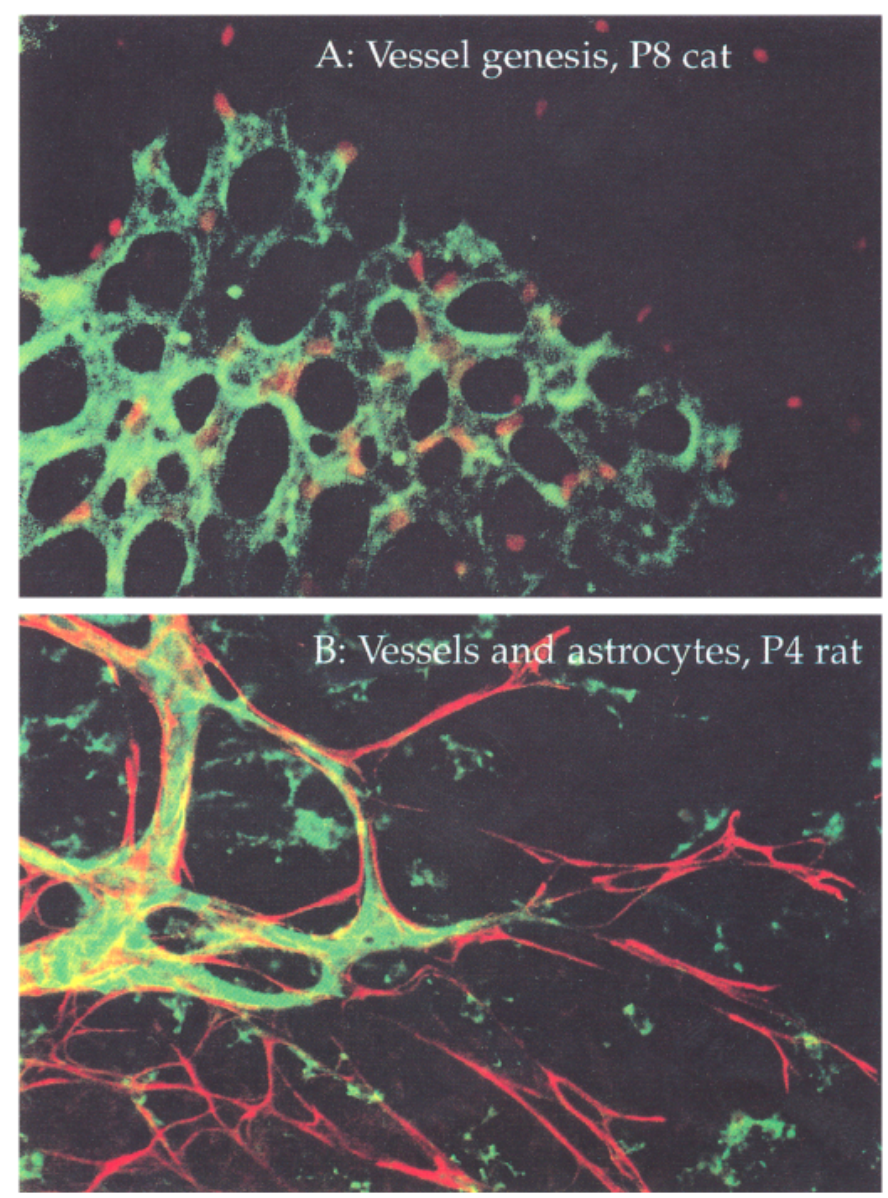

Figure 2. Cellular detail of the superficial layer of vessels. A, This image, obtained by confocal microscopy, shows the edge of the superficial layer in a P8 cat. The vessels are actively extending towards the margin of the retina (to the top and right of the plate). The vessels are labeled by fluorescein bound to the lectin $G$. simplicifolia. Dividing cells are labeled by TRITC bound to an antibody to BrdU (see Materials and Methods). $B$, At the edge of the spreading superficial vessels in a P4 rat, astrocytes (labeled by TRITC conjugated to an anti-GFAP antibody) spread ahead (to the right) of the newly forming vessels, which are labeled with FITC-conjugated $G$. simplicifolia lectin (see Materials and Methods).

the junction of the inner nuclear and outer plexiform layers. At this level, they extend branches parallel to the retinal surface, to form the deep layer of the retinal vasculature (Ashton and Blach, 1961). The outer half of the retina, comprising the outer nuclear and receptor layers, remains avascular. In the Sabra rat, the first radially extending vessels were detected at $\mathrm{P} 4$, at the edge of the optic disk. By P7, such vessels could be detected over a considerably wider area of retina, and the first elements of the deep plexus were apparent near the optic disk. The process spreads peripherally and reaches the edge of the retina at approximately P14. In a retina aged between P1 and P10, therefore, a gradient of vascular development can be traced between the optic disk and the retinal margin. In the cat the retinal vessels form in a similar pattern, the formation of the superficial vessels lasting from embryonic day E52 to P30, and of the deep layer from P10 to P30 (Chan-Ling et al., 1990).

Figure 2 shows cellular detail at the edge of the vessels forming at the inner surface of the retina. In Figure $2 A$ the green fluorophore, conjugated to the lectin, shows the most peripheral, and hence newest formed vessels in a P8 cat retina. Scattered microglial cells are also labeled by the lectin. The red fluorophore is conjugated to BrdU which was injected $1 \mathrm{hr}$ before fixation, and taken up by cells in S-phase of mitosis. Labeled (dividing) nuclei are numerous in the newly forming vessels. The BrdU-labeled nuclei located peripheral to the vessels are the nuclei of either vascular precursor cells (Chan-Ling et al., in press, a,b) or astrocytes. The peripheral limit of the spreading superficial vessels is sharp and the most peripheral endothelial cells extend processes towards avascular retina. In Figure $2 B$. The red label (conjugated to an anti-GFAP antibody) shows astrocytes spreading across the $\mathrm{P} 4$ rat retina. Astrocytes migrate into the retina from the optic disc (Ling and Stone, 1988; Watanabe et al., 1988; Ling et al., 1989) at approximately the same time as the vessels form, always spreading ahead of the vessels. As the astrocytes spread they form a spaced array, shaped by the astrocyte-astrocyte interaction termed contact-spacing (Chan-Ling et al., 1991; Tout et al., 1993b), in which the somas of astrocytes remain separate, but neighboring astrocytes maintain contact via their processes. Figure $2 B$ shows that new vessels form along the processes of the astrocytes. The tight association between astrocytes and newly formed capillaries may be the result of chemoattraction of endothelial cells (or of their presumed precursors) to astrocytes. Alternatively, it may reflect the ability of astrocytes to induce the contact-dependent formation of capillary-like structures (Laterra et al., 1990).

The deep layer of vessels forms by a process of budding from vessels of the superficial layer, without being preceded by populations of either vascular precursor cells (Chan-Ling et al., 1990) or astrocytes (Chan-Ling et al., 1991).

Transient expression of VEGF by astrocytes precedes vessel formation at the inner surface. In the P1 rat VEGF was expressed in the neural retina, by cells adjacent to the inner limiting membrane, near the optic disk. At high magnification it was apparent that VEGF expression was localized to particular cell bodies near the limiting membrane. VEGF was also expressed, throughout the period under study, in the retinal pigment epithelium (RPE); this expression does not appear to be a factor in the formation of the retinal vasculature.

Over the next several days, the VEGF-expressing cells at the inner surface spread progressively towards the margin of the retina, and VEGF expression in more central retina was downregulated. For example, in a P3 retina, expression of VEGF is already less in cells near the optic disk than in cells closer to the retinal margin (Fig. 3B). The superficially located, VEGFexpressing cells reached the edge of the rat retina at about P7.

In the cat, as in the rat, the first expression of VEGF occurred in superficially located cells which spread across the retina. By P4 VEGF-expressing cells had reached approximately $2 \mathrm{~mm}$ from the optic disk (data not shown), and at P10 they had reached $8 \mathrm{~mm}$ from the disk. At P10 moreover, VEGF expression in more central retina had been downregulated (see Fig. $6 A)$.

In both rat and cat, the VEGF-expressing cells extended further from the optic disk than the developing blood vessels (Fig. 3; see also Figs. 6, 8). This relationship persisted until first the VEGF-expressing cells, and then the vessels, reached the margin of the retina.

The VEGF-expressing cells at the inner surface were identified as astrocytes because (1) they lie internal to the ganglion cells (Fig. 3C), at the level characteristic of retinal astrocytes (Stone and Dreher, 1987; Ling et al., 1989); (2) they spread 

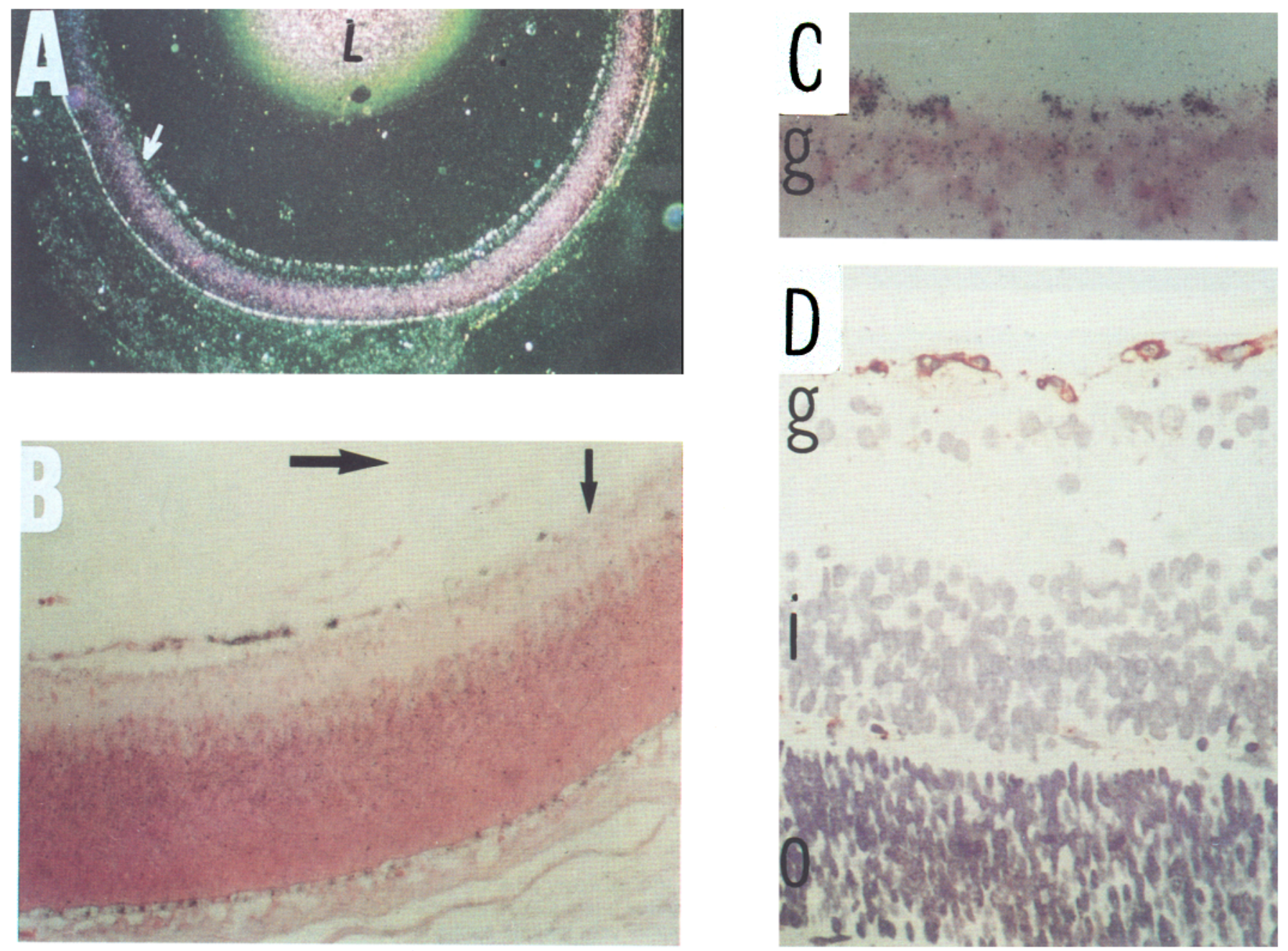

Figure 3. VEGF expression at the inner surface of the retina. A, A dark-field image of A P1 rat retina hybridized with a VEGF-specific probe. White arrow indicates the extent of astrocyte spreading, as determined by inspection at high power of the VEGF-hybridized, hematoxylin-stained sections, in which astrocytes are seen as darkly stained, bipolar cells spreading very superficially in the axon layer, ahead of the vessels. Note that VEGF-expressing cells reside in the innermost retinal layer (presumably astrocytes) as well in the outermost retinal layer (the retinal pigmented epithelium). B, P3 rat retina. The horizontal arrow indicates the direction of vessel extension, and the vertical arrow indicates the extent of astrocyte spreading. Note the nonuniform distribution of VEGF-expressing cells in the astrocyte cell level, as compared with the uniform distribution of VEGF-expressing cells in the retinal pigmented epithelium. $C$ and $D$, High magnification view of a peripheral region in a P10 retina. Serial sections were either hybridized with a VEGF-specific probe $(C)$ or stained with GFAP $(D)$. At this stage, the innermost cellular layer, composed mostly of vessels and astrocytes (the GFAP+ cells), is clearly distinguishable from the underlying ganglion cell layer, allowing the identification of VEGFexpressing cells as astrocytes. $g$, Ganglion cells; $i$, inner nuclear layer; $o$, outer nuclear layer.

across the retina at the time and in the pattern previously described for astrocytes (cat: Ling and Stone, 1988; rat: above and Ling et al., 1989); and (3) comparable cells in adjacent sections label with an antibody for the astrocyte-specific glial fibrillary acidic protein (GFAP) (Fig. 3D).

Transient expression of VEGF by presumed Müller cells precedes vessel formation at the INL. In rats up to P5, the INL had not yet separated from the cytoblast layer, and specific expression of VEGF was restricted to the inner surface. By P7, the INL had formed near the optic disk, and VEGF expression was apparent in the inner nuclear layer, with maximal level of expression within $0.5 \mathrm{~mm}$ of the disc and a lower level of expression along the next $1 \mathrm{~mm}$ of the retina (data not shown). In the P10 rat, VEGF expression in the INL was very strong and had extended towards the retinal margin (Fig. 4A). The VEGF signal was concentrated in a stratum of cells at approximately the mid- dle of the thickness of the layer. The VEGF signal in the INL appears punctate at high magnification (Fig. 4B).

Along the radial axis, VEGF expression in the INL moved gradually from the optic disk to the retina margin. At P7 expression could be detected only at the optic disk (not shown). By P10, expression had faded at the optic disk, was very strong in the mid-periphery and had not yet reached the margin (Fig. 4 ); and by P14 had faded from the mid-periphery and was confined to the peripheral $1-2 \mathrm{~mm}$ of retina (not shown). This pattern of spread closely precedes the formation of the deep plexus of retinal vessels (Fig. 1).

The VEGF-producing cells in the INL are tentatively identified as Müller cells because (1) their somas are located in a substratum of the INL, at approximately the middle of the layer, known to be occupied by Müller cells; (2) their somas are closely adjacent, and are distributed apparently uniformly between 

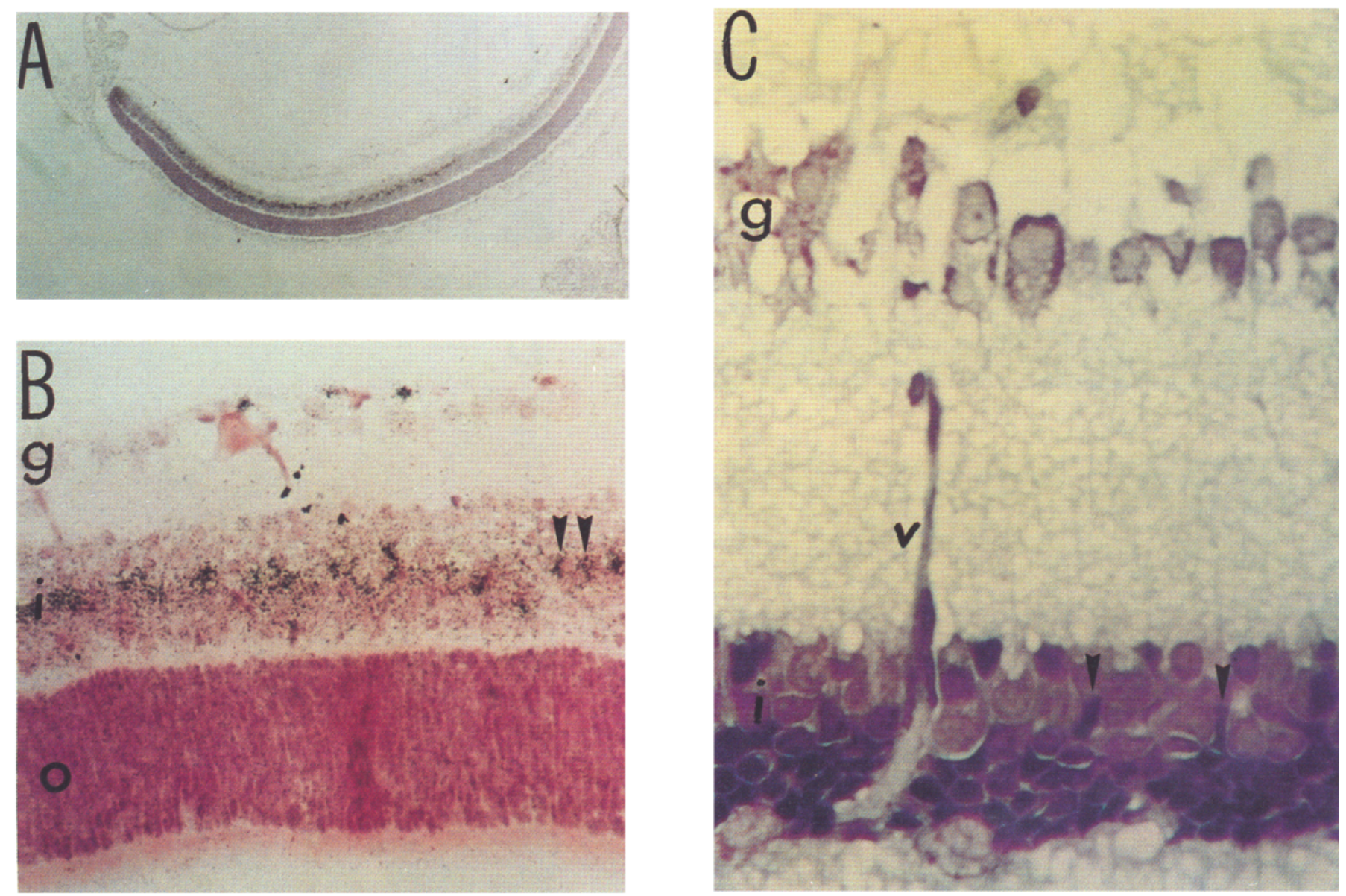
Figure 4. VEGF expression in the inner nuclear layer. Sections of a P10 rat retina. A, A low magnification image showing that, within the INL,
the level of VEGF expression in the periphery is significantly higher than expression in areas closer to the optic disk (to the right side of the picture). $B$, A higher magnification of the former, showing VEGF-producing cells residing around the midline of the INL. Certain positive cells are marked by arrows to emphasize the punctate pattern of VEGF-expressing cells. $C$, A serial thin $(3 \mu \mathrm{m})$ section stained with hematoxylin and eosin, showing further details, including Muller cells (indicated by arrows) and a descending vessel $(v)$.

the center and the periphery of the retina, a distribution characteristic for Müller cells; and (3) in adjacent thin sections cut to allow discrimination between the cell classes of the INL, the somas of Müller cells can be identified at the same level within the INL, and in a similar punctuate spacing pattern, to VEGFpositive cells (Fig. 4C).

No other expression of VEGF was noted within the developing neural retina. VEGF expression by the retinal pigment epithelium was a feature of the developing retina throughout the period examined, in both species.

Summary and hypothesis. Figure 5 summarizes VEGF expression in the developing retina of the cat and rat. The developmental ages of the retina shown summarize data available for the rat (Braekevelt and Hollenberg, 1970; Webster and Rowe, 1991) and cat (Donovan, 1966; Rapaport and Stone, 1984). In both species, the first expression of VEGF in neural retina is seen in astrocytes at the inner surface. As the astrocytes spread across the retina, VEGF expression is strong in the most peripheral astrocytes, and falls in those closer to the disk. By P10 in the rat, VEGF expression in this superficial layer is restricted to the edge of the retina, and by P14 has disappeared. A second region of VEGF expression appears in the INL, with some delay after the astrocyte expression. This expression also spreads peripherally and fades centrally, so that VEGF expression is pro- gressively limited to the peripheral retina, and then disappears. In each case the wave of VEGF expression seems closely to precede the formation of vessels. The expression of VEGF by RPE, is also shown in Figure 5. It occurs some distance from the formation of the retinal vasculature, and close to the choroid.

We hypothesize (see Discussion) that the astrocytes spreading across the retinal surface function as detectors of hypoxia in the inner layers of retina and, in response to hypoxia, secrete VEGF, which causes vessel growth. By bringing oxygen, vessel function causes a downregulation of VEGF expression, limiting the hypoxic stimulus. We suggest that Müller cells function as detectors of hypoxia in the middle layers of retina and in response secrete VEGF, which causes the growth of the deep layer of retinal vessels, whose function then limits the hypoxic stimulus. The initial growth of these vessels is radial because, we suggest, it follows the radial orientation of the Müller cells. Finally, we postulate that the expression of VEGF by the RPE is not related to formation of the retinal vasculature, but to the choroidal circulation. We tested these hypotheses in three ways.

Developing retinal vessels express flk-1 VEGF receptors. If VEGF acts as an endothelial growth factor in developing retina, its receptors should be expressed by the developing vessels. We therefore hybridized adjacent sections with the respective probes. Figure $6 A$ shows VEGF expression in the mid-periphery 


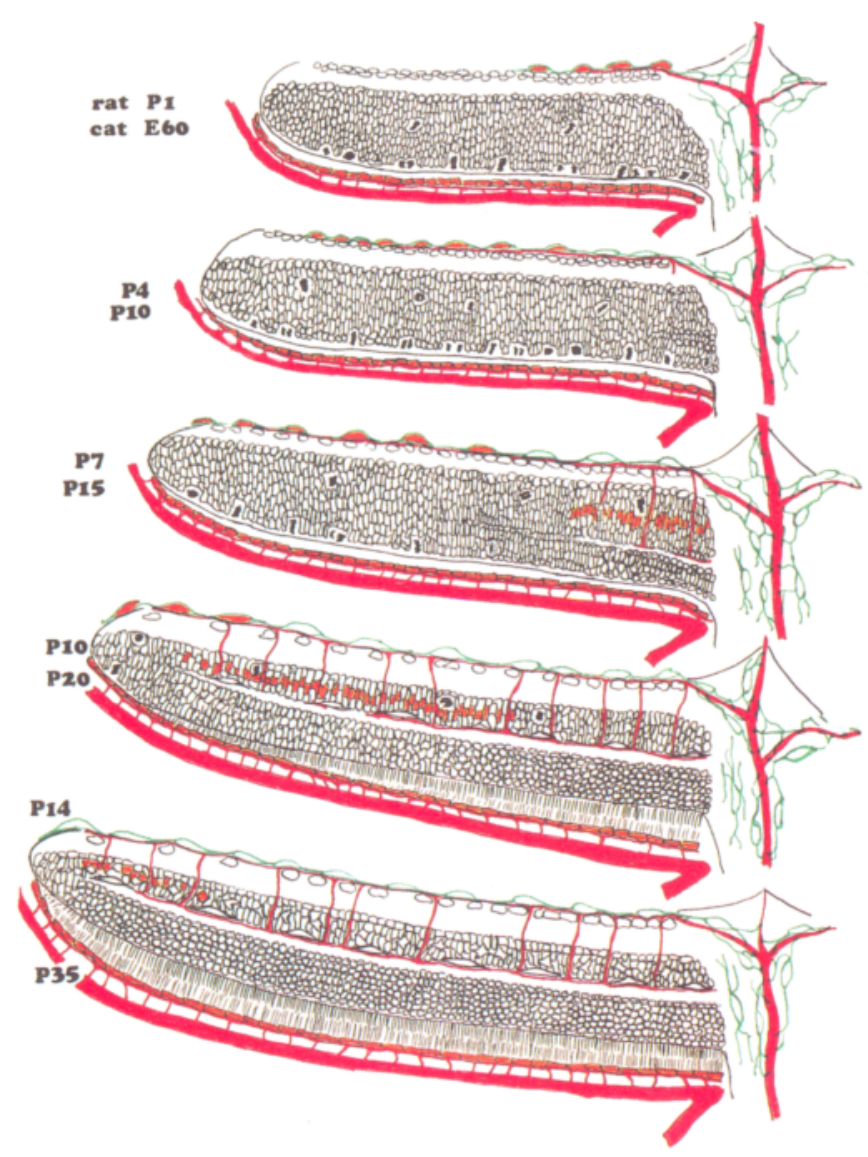

Figure 5. Summary diagram of VEGF expression in the developing retina. Each drawing represents a section of the retina from the optic disk (at right) to one margin of the retina (at left). The five drawings form a developmental series applicable to the rat and cat; the ages of the two species represented in each diagram are shown at left. Rat Pl/ cat E60, The neural retina is comprised of two cellular layers. The outer (cytoblast) layer is still generating neurones, and mitotic figures are numerous at the outer surface of the neural retina, adjacent to the pigment epithelium. The inner (ganglion cell) layer contains postmitotic neurones which have already separated from the cytoblast layer. The choroid circulation is well formed, and the hyaloid artery extends through the optic disk to supply the vitreous body and the lens. Astrocytes (in green) have begun to migrate across the surface of the retina, and the superficial layer of vessels has begun to form. At this age and subsequent ages, VEGF (in orange) is expressed strongly in the RPE. At this age, VEGF is also expressed in the neural retina, by the astrocytes. Rat P4/cat P10, The spread of astrocytes and superficial vessels has continued, and the first "descending" vessels have begun to bud from the superficial layer. VEGF expression is strong in the most peripheral astrocytes, but has faded in astrocytes near the optic disk. Rat $P 7 /$ catP15, The separation of the cytoblast into the inner and outer nuclear layers, by the formation of the outer plexiform layer, has begun near the optic disk; where this separation has taken place, cell division has halted. Astrocytes have reached the edge of the retina, the most peripheral of them expressing VEGF, and the superficial layer of vessels has spread correspondingly. Expression of VEGF is now apparent in the INL and, correspondingly, some descending vessels have reached the level of the deep vascular plexus, near the optic disk. Rat P10/cat $P 20$, The division of the retina into its layers is almost complete, mitosis in the neural retina has almost ceased, and the formation of the inner and outer segments of the receptors has commenced, beginning near the optic disk. VEGF expression at the inner surface is restricted to the most peripheral margin of the retina, and the superficial vasculature is close to the edge of the retina. VEGF expression in the INL is now strong in the mid-periphery and, accordingly, the deep vascular plexus has spread peripherally. Rat PI4/cat P35, The formation of the neural retina and its vasculature is essentially complete. VEGF expression by region of a P10 cat retina. Confirming the above results, VEGF expression is strongest in astrocytes at and just peripheral to the leading vessels, whereas, more centrally, VEGF expression by astrocytes has diminished. Figure $6 C$ shows in an adjacent section that $f k-1$ is expressed where the superficial vessels are forming and that the zone of $f k-l$ expression partly overlaps with, but lags behind, the zone of VEGF expression, matching precisely the extent of vessel spread. At high magnification (Fig. $6 D$ ) the expression of $f k-1$ was seen to be confined to endothelial cells in formed vessels.

Hypoxia induces VEGF expression in glial cells in vitro. If macroglial cells express VEGF in response to hypoxia, their response to hypoxia should be demonstrable in the absence of other cell classes. We therefore compared steady state levels of VEGF mRNA in primary cultures of astrocytes and in C6 glioma cells grown in normoxia and hypoxia. Initial experiments (data not shown) used a Northern blot analysis to confirm our earlier observation (Shweiki et al., 1992) that exposure to hypoxia augments VEGF expression by glioma cells. To identify particular species of VEGF mRNA induced by hypoxia, we carried out a quantitative reverse transcriptase-PCR analysis, using VEGF-specific primers corresponding to external exons (i.e., using oligonucleotide primers that anneal with all the known, alternatively spliced VEGF mRNAs).

Figure 7 shows the results of this analysis. In both astrocytes and C6 glioma cells, exposure to hypoxia led to augmented expression of mRNAs encoding $\mathrm{VEGF}_{188}, \mathrm{VEGF}_{164}$, and $\mathrm{VEGF}_{120}$, with no apparent change in stoichiometry. At least the two smaller mRNA species encode a secreted protein (Ferarra et al., 1992).

Hyperoxia in vivo suppresses vessel formation and VEGF expression selectively in neural retina. If tissue oxygen levels control VEGF expression by retinal macroglia, then VEGF expression should be suppressed by exposing the retina to hyperoxia. Rat pups were raised from P1-P3, and from P8-10, in high levels of oxygen (70-80\%), levels known to increase retinal oxygen tension (Linsenmeier and Yancey, 1989; Alder et al., 1990) and inhibit vessel growth in the rat. Figure 8, $E$ and $F$, compares the superficial vasculature of a P10 rat raised from P8 to P10 in $70-80 \%$ oxygen $(F)$ with that of a littermate control $(E)$. Confirming earlier reports in both rat and cat (Ashton and Blach, 1961; Ashton, 1966, 1968; Phelps, 1990; Chan-Ling et al., 1992a), hyperoxia caused the obliteration of many capillaries near the optic disk (not shown) and, where capillaries persist, increased the width of the capillary free zones which flank arteries and the mesh size of surviving capillaries. In addition, the formation of deep vasculature, which normally has begun by P10 (above) was completely suppressed by the hyperoxia.

Figure 8 compares the expression of VEGF in the retinas of rats exposed to hyperoxia with littermate controls. At P3, VEGF is strongly expressed by the leading astrocytes at the inner surface of the normoxic retina (Fig. $8 A$ ); in a littermate kept in 70$80 \%$ oxygen for $2 \mathrm{~d}$ prior to fixation, the VEGF signal was significantly downregulated (Fig. 8B). At P10, VEGF expression is strong in the middle of the INL of a normoxic retina. Again, expression was markedly weaker in the retina kept in $70-80 \%$ oxygen for $2 \mathrm{~d}$ prior to fixation (Fig. $8 D$ ). A similar result was

$\leftarrow$

astrocytes has subsided, and expression in the INL is restricted to the peripheral margin of the retina. 

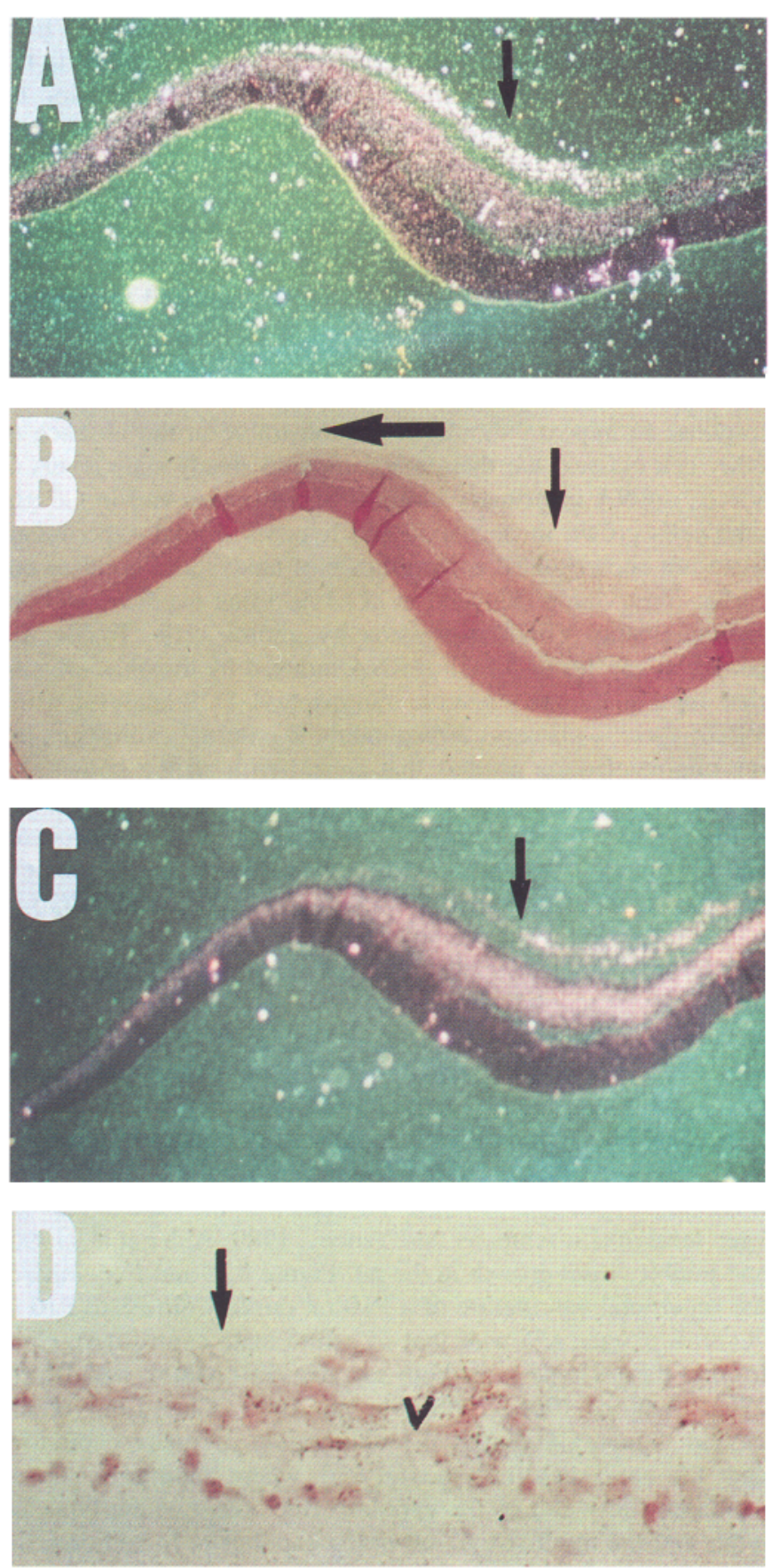

Figure 6. Spatial relationship of cells expressing VEGF and cells expressing VEGF receptors $(f k-1)$. Serial sections of a P10 cat retina were hybridized with a VEGF-specific probe $(A)$ and a VEGF receptor (KDR/ $f(k-1)$-specific probe $(B-D)[B$ and $C$ are low magnification, bright-field and dark-field images, respectively. $D$ is a higher magnification of the ganglion cell and optic fiber layers, showing sections through vessels $(v)$. Horizontal arrow points at the direction of vessel spreading, and the vertical arrows point at the leading edge of vessels.

obtained in the cat. Specifically, in the retinas of a cats aged P4 exposed to hyperoxia (70-80\%) for $24 \mathrm{hr}$ and $72 \mathrm{hr}$ before fixation, the normal expression of VEGF by astrocytes at the inner surface of the retina was eliminated (data not shown).

The hypothesis that VEGF expression in the RPE is not related to the formation of the retinal vasculature was also tested

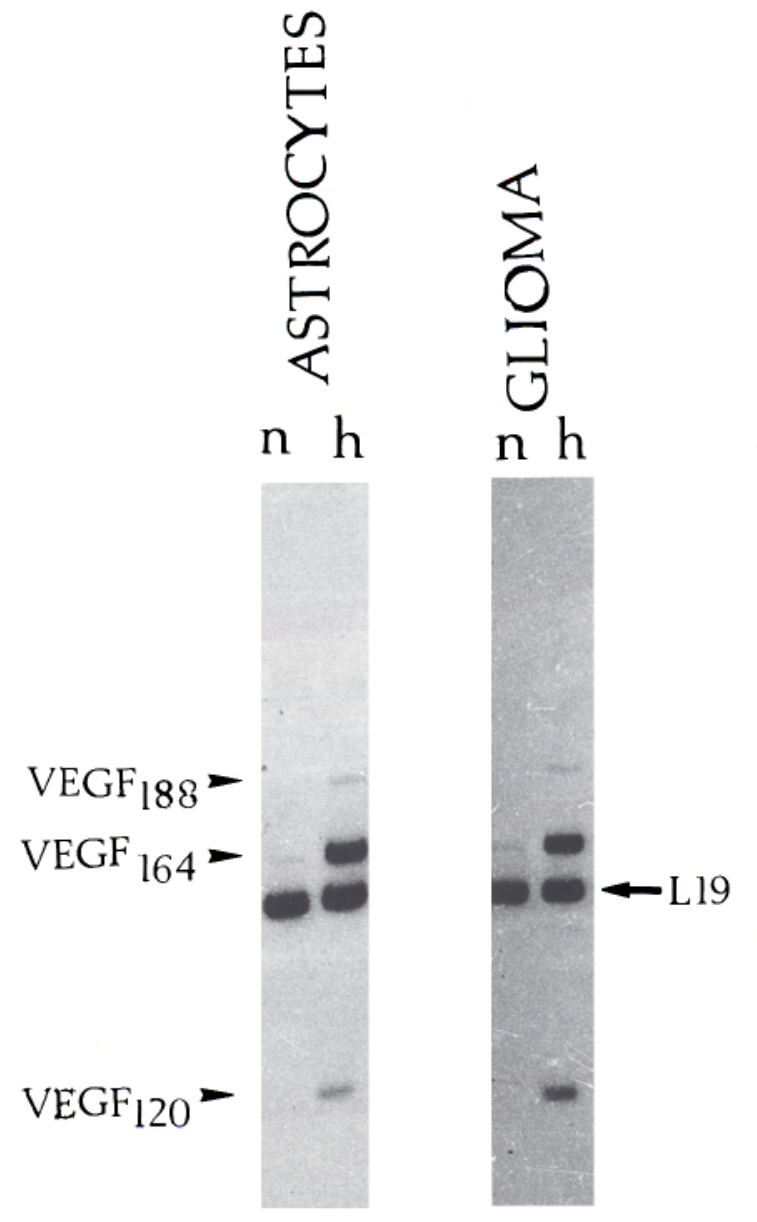

Figure 7. Reverse transcriptase-PCR analysis of VEGF mRNA in primary astrocytes and glioma cultures. See Materials and Methods for description of cells and experimental procedures. Amplified VEGF cDNA fragments (indicated by arrowheads) are $297 \mathrm{bp}, 225 \mathrm{bp}$, and $99 \mathrm{bp}$ long (encoding $\mathrm{VEGF}_{188}, \mathrm{VEGF}_{164}$, and $\mathrm{VEGF}_{120}$, respectively). Leftward arrow points at the 194 bp long fragment of coamplified ribosomal L19 cDNA. $n$, Cells grown under normoxia; $h$, cells grown under hypoxia.

by this experiment. Specifically, the VEGF signal in the RPE was unaffected by hyperoxia in the P3 and P10 rats (Fig. 8A$D$ ), and in the $\mathrm{P} 4$ cat material described above, even though the hyperoxia suppressed both the expression of VEGF in the neural retina, and the formation retinal vessels.

\section{Discussion}

The expression of VEGF during normal retinal development has been described from in situ hybridization studies of the retina of neonatal rats and cats. VEGF is expressed in two layers of developing neural retina, at its inner surface and, after a delay, in the middle of the inner nuclear layer. Our observations suggest that VEGF is expressed selectively by the macroglia of the retina, by astrocytes at the inner surface and by Müller cells in the inner nuclear layer. The expression of VEGF in the inner layer closely precedes the formation of the superficial layer of retinal vessels, while its expression in the inner nuclear layer closely precedes the formation of the deep layer of retinal vessels.

Because of the close correlation in topography and timing between VEGF expression and vessel growth, we hypothesized that VEGF functions as a vasoformative growth factor, secreted 

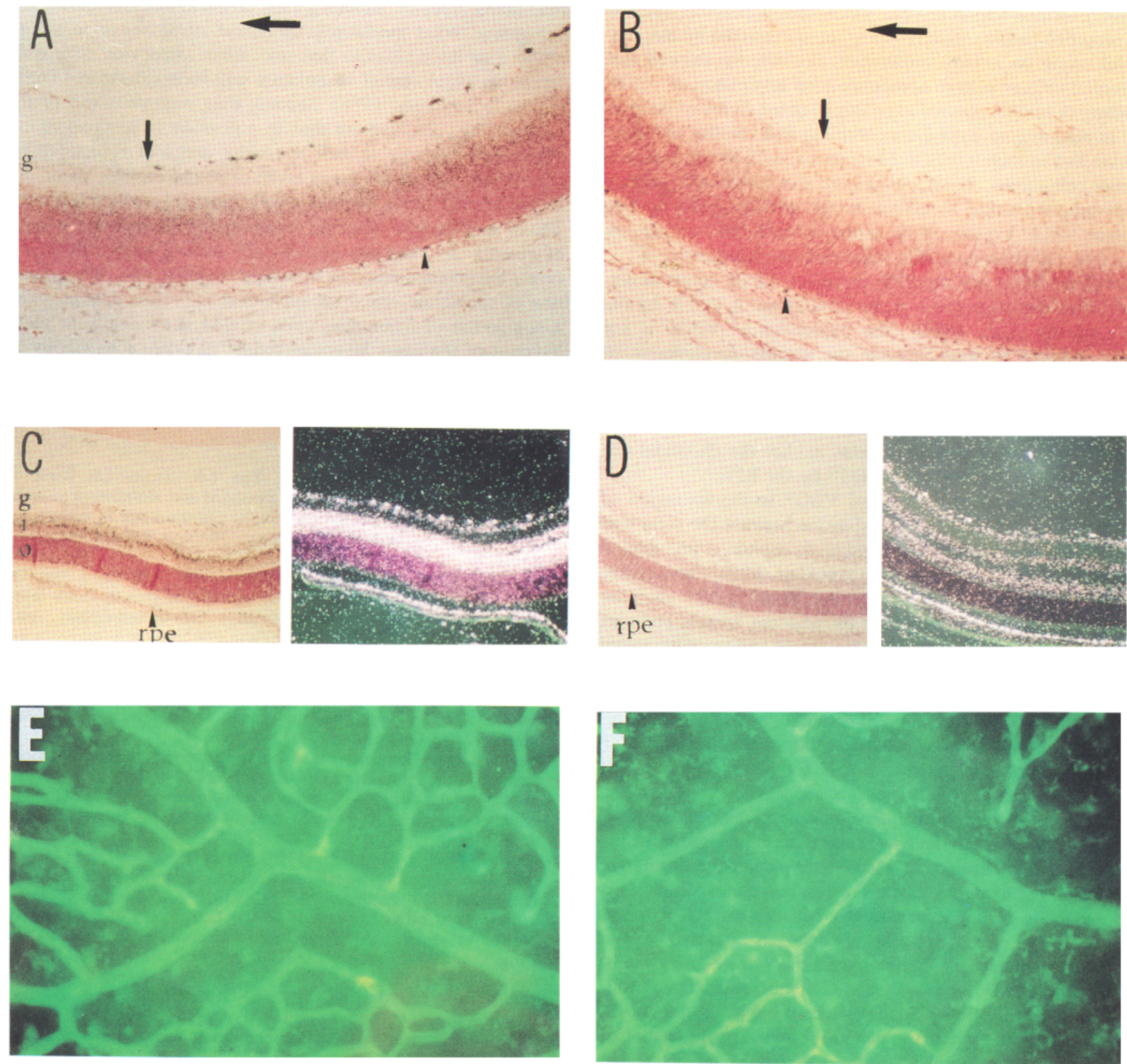

Figure 8. Effect of hyperoxia on VEGF expression and vessel formation in the intact retina. $A$ and $B$, VEGF expression in the most peripheral astrocytes of P3 rat littermates raised in normoxia $(A)$ and in hyperoxia for $2 \mathrm{~d}(B)$. Horizontal arrows indicate the direction of vessel spreading. Vertical arrows indicate the extent of astrocyte spreading. Small arrowheads point at VEGF-expressing retinal pigmented epithelium (RPE) cells. $C$ and $D$, VEGF expression in the INL of P10 rat littermates raised in normoxia $(C)$ and in hyperoxia for $2 \mathrm{~d}(D)$. Sections shown in $A-D$ were processed, hybridized, and exposed in parallel. $E$ and $F$, Effect of hyperoxia on vessel growth in rat retina. The left panel shows a region of the superficial layer of vessels in the retina of a P3 animal raised in normoxia. The right panel shows a comparable region from the retina of a littermate aged $\mathrm{P} 3$ but raised from $\mathrm{P} 1$ in $70-80 \%$ oxygen.

by the retina to control the formation of its vessels. The function of such a factor in the development of retinal vessels was proposed in specific terms by Michaelson (1954). Because hypoxia is an important stimulus for the development of retinal vessels (Ashton, 1966; Phelps, 1990; Chan-Ling and Stone, 1993), and because VEGF expression is known to be induced by hypoxia (Plate et al., 1992; Shweiki et al., 1992; Menchenko et al., 1994), we hypothesized that retinal macroglia express VEGF in response to levels of tissue hypoxia which occur normally during development. Such hypoxia (termed "physiological hypoxia" by
Chan-Ling and Stone, 1993) develops, these authors suggest, when increasing retinal metabolism limits the flow of oxygen from the choroid to the inner layers of the retina. The expression of VEGF by the retinal macroglia is transient, and we suggest that expression is limited by the function of the newly formed vessels, in bringing blood-borne oxygen. In this way the formation of vessels is matched to the metabolic activity of developing neurones.

We have tested this model in three ways. First, we demonstrated that the high affinity $f k-1$ receptor for VEGF is expressed 
by newly formed retinal vessels. VFGF thus appears to function in a paracrine fashion in developing retina, secreted by macroglia and acting on nearby vasculature. Second, we showed that macroglial cells in culture upregulate their expression of VEGF, including of mRNA species encoding secreted forms of the protein, in response to hypoxia, confirming their ability to detect hypoxia and to secrete VEGF in response, without the presence of other cell classes. Third, we showed that levels of hyperoxia which suppress vessel formation in the developing retina also suppress VEGF expression by astrocytes and Müller cells, respectively. All three tests thus supported our hypotheses.

Our observation that the RPE expresses VEGF confirms earlier in vitro demonstrations of the production of $\mathrm{VEGF}$ by the RPE (Adamis et al., 1993). This expression of VEGF is not related spatially or temporally to the normal development of retinal vessels, and may be important for the formation of the choroidal circulation. In accordance with this suggestion, hyperoxia, which inhibits both vessel formation and VEGF expression in the neural retina, affects neither the choroidal circulation, nor the expression of VEGF by the RPE.

The above model of the role of VEGF in the formation of vessels was explored in the neural retina because the failure of the choroidal circulation to autoregulate makes it possible to create hyperoxia in the retina by exposing animals to oxygenenriched atmospheres (Chan-Ling and Stone, 1993). In other parts of the CNS, autoregulation by adjacent vessels prevents both obliteration of vessels during hyperoxia and any consequent proliferative vasculopathy (Ashton, 1968). Nevertheless, the main features of the model may prove general to the CNS. A role for VEGF in the formation of cerebral vasculature was suggested by recent studies which relate the expression of VEGF and its receptors to patterns of vessel formation (Breier et al., 1993; Millauer et al., 1993; Peters et al., 1993).

Tissue oxygen tension as a developmental signal in the CNS. There is evidence from the adult central nervous system to support the idea that tissue metabolism determines the formation of vasculature. For example, patches of cerebral cortex which show high metabolic activity, such as the whisker barrels of rodent somatosensory cortex, or the cytochrome oxidase-positive "puffs" of primate visual cortex have a relatively dense vascular supply (Patel, 1983; Borowsky and Collins, 1989; Zheng et al., 1991; Riddle et al., 1993). Such patches of high vascular density could be produced by regional variations in neuronal metabolism during normal development, by the mechanism set out above. The concept of "physiological hypoxia" suggested as the normal stimulus for retinal vascularization may also explain regional variations in the vascular supply of the normal brain.

Our in vitro observations (Fig. 8) provide evidence the glial cells can respond to hypoxia in the absence of other cell classes. The mechanisms by which glial cells might detect hypoxia remain unknown, although similarity has been described between the oxygen sensing mechanisms regulating the expression of VEGF and of erythropoietin (Goldberg and Schneider, 1994).

Why two classes of retinal macroglia? Stone and Dreher (1987) noted an enigma in the glial organization of the mammalian retina. The retina generates its own macroglia, the radially oriented Müller cells. Müller cells share many properties of astrocytes, including the ability to invest axons and form the glia limitans of the retina and of vessels (Holländer et al., 1991), to induce the formation of barrier properties in capillaries (Janzer and Raff, 1987; Tout et al., 1993a), and to express VEGF in response hypoxia (above). Nevertheless, astrocytes enter certain mammalian species, including rats, cats, and humans, during development. What special role do retinal astrocytes perform?

We note three clues to that role. First, astrocytes enter only retinas in which retinal vasculature will form (Stone and Dreher, 1987; Schnitzer, 1988). Second, they migrate ahead of the vessels (Fig. $2 B$ ), and are thus in a position to respond to environmental signals in still avascular areas of retina. Third, their restriction to the inner layer of retina enables them, we argue, to respond to hypoxia of the inner layers by expressing VEGF, and thereby inducing formation of the superficial layer of vessels. By contrast, Müller cells extend across all retinal layers and cannot respond in a layer-specific way. The migration of astrocytes across the surface of the retina thus provides a population of macroglial cells located strategically to sense hypoxia of the inner layers of retina, to cxpress VEGF in response, thereby to induce the formation of the superficial layer of retinal vessels.

\section{References}

Adamis AP, Shima DT, Yeo K-T, Yeo T-S, Brown LF, Berse B, D'Amore PA, Folkman J (1993) Synthesis and secretion of vascular permeability factor/vascular endothelial growth factor by human retinal pigment epithelial cells. Biochem Biophys Res Commun 193: 631-638.

Adamis AP, Miller JW, Bernal MT, d'Amico DJ, Folkman J, Yeo TK, Yeo KT (1994) Increased vascular endothelial growth factor levels in the vitreous of eyes with proliferative diabetic retinopathy. Am J Ophthalmol 118:445-450.

Alder VA, Ben-Nun J, Cringle SJ (1990) $\mathrm{PO}_{2}$ profiles and oxygen consumption in cat retina with an occluded retinal circulation. Invest Ophthalmol Visual Sci 31:1029-1034.

Ashton N (1966) Oxygen and the growth and development of retinal vessels. Am J Ophthalmol 62:412-435.

Ashton N (1968) Some aspects of the comparative pathology of oxygen toxicity in the retina. Br J Ophthalmol 52:202-531.

Ashton N, Blach R (1961) Studies on developing retinal vessels. VIII. effect of oxygen on the retinal vessels of the ratling. Br J Ophthalmol 45:321-340.

Ashton N, Ward B, Serpell G (1954) Effect of oxygen on developing retinal vessels with particular reference to the problem of retrolental fibroplasia. Br J Ophthalmol 38:397-430.

Borowsky IW, Collins RC (1989) Metabolic anatomy of brain; a comparison of regional capillary density, glucose metabolism, and enzyme activities. J Comp Neurol 288:401-413.

Braekevelt CR, Hollenberg MJ (1970) The development of the retina of the albino rat. Am J Anat 127:281-302.

Breier G, Albrecht U, Sterrer S, Risau W (1992) Expression of vascular endothelial growth factor during embryonic angiogenesis and endothelial cell proliferation. Development 114:52-532.

Chan-Ling T, Stone J (1991) Factors determining the morphology and distribution of astrocytes in the cat retina: a 'contact-spacing' model of astrocyte interaction. J Comp Neurol 303:387-399.

Chan-Ling TL, Stone J (1992) Degeneration of astrocytes in feline retinopathy of prematurity causes failure of the blood-retinal barrier. Invest Ophthalmol Visual Sci 33:2148-2159.

Chan-Ling T, Stone J (1993) Retinopathy of prematurity: its origins in the architecture of the retina. Prog Retinal Res 12:155-178.

Chan-Ling T, Halasz P, Stone J (1990) Development of retinal vasculature in the cat: processes and mechanisms. Curr Eye Res 9:459478

Chan-Ling T, Tout S, Holländer H, Stone J (1992) Vascular changes and their mechanisms in the feline model of retinopathy of prematurity. Invest Ophthalmol Visual Sci 33:2128-2147.

Chan Ling T, Gock B, Stone J (in press, a) 'Physiological hypoxia' is the stimulus for normal retinal vasculogenesis. Invest Ophthalmol Visual Sci, in press.

Chan-Ling T, Gock B, Stone J (in press, b) Supplemental oxygen therapy: basis for non-invasive treatment of retinopathy of prematurity. Invest Ophthalmol Visual Sci, in press.

Chen $\mathrm{C}-\mathrm{H}$, Chen $\mathrm{SC}$ (1980) Angiogenic activity of vitreous and retinal extract. Invest Ophthalmol Visual Sci 19:596-602. 
Chen C-II, Chen SC (1984) Mediation of vascular endothelial proliferation by factors in fetal bovine retinal extracts and serum. Exp Eye Res 39:469-478.

Chen $\mathrm{C}-\mathrm{H}$, Chen SC (1987) Evidence of the presence of a specific vascular endothelial growth factor in fetal bovine retina. Exp Cell Res 169:287-295.

Connolly DT (1991) Vascular permeability factor: a unique regulator of blood vessel function. J Cell Biochem 47:219-223.

D'Amore PA, Glaser BM, Rrunson SK, Fenselau AH (1981) Angiogenic activity from bovine retina: partial purification and characterization. Proc Natl Acad Sci USA 78:3068-3072.

Donovan $A$ (1966) The postnatal development of the cat's retina. Exp Eye Res 5:249-254.

Ferrara N, Houck KA, Jakeman LB, Winer J, Leung DW (1991) The vascular endothelial growth factor family of polypeptides. J Cell Biochem 47:211-218.

Ferrara N, Houck KA, Jakeman LB, Leung DW (1992) Molecular and biological properties of the vascular endothelial growth factor family of proteins. Endocr Rev 13:18-32.

Frangakis MV, Kimelberg HM (1984) Dissociation of neonatal rat brain by dispase for the preparation of primary astrocyte cultures. Neurochem Res 9:1689-1698.

Glaser BM, D'Amore PA, Mechels RG, Patz A, Fenslau A (1980) Demonstration of vasoproliferative activity from mammalian retina. J Cell Biol 84:298-304.

Goldberg MA, Schneider TJ (1994) Similarities between the oxygensensing mechanisms regulating the expression of vascular endothelial growth factor and erythropoietin. J Biol Chem 269:4355-4369.

Gospodarowitz D, Abraham JA, Schilling J (1989) Isolation and characterization of vascular endothelial mitogen produced by pituitaryderived follicular stellate cells. Proc Natl Acad Sci USA 86:73117315.

Holländer H (1992) Development of the mammalian retina. Commun Dev Neurohiol 1:341-358.

Holländer H, Makarov F, Dreher Z, van Driel D, Chan-Ling T, Stone J (1991) Functions of the macroglia of the retina: the sharing and division of labour between astrocytes and Müller cells. J Comp Neurol 313:587-603

Jakeman LB, Armanini M, Phillips HS, Ferrara N (1993) Developmental expression of binding sites and messenger ribonucleic acid for vascular endothelial growth factor suggests a role for this protein in vasculogenesis and angiogenesis. Endocrinology 133:848-859.

Janzer RC, Raff MC (1987) Astrocytes induce blood-brain barrier properties in endothelial cells. Nature 325:253-257.

Kim K.J, I. B R, Winer .I, et al. (1993)Inhibition of vascular endothelial growth factor-induced angiogenesis suppresses tumor growth in vivo. Nature 362:841-844.

Knighton DR, Hunt TK, Schcucnstuhl H, Holliday BJ, Wcrb Z, Banda $M$ (1983) Oxygen tension regulates the expression of angiogenesis factor by macrophages. Science 221:1283-1285.

Laterra J, Guerin C, Goldstein E (1990) Astrocytes induce neural microvascular endothelial cells to form capillary-like structures in vitro. J Cell Physiol 144:204-215.

Ling TC, Stone J (1988) The development of astrocytes in the cat retina: evidence of migration from the optic nerve. Dev Brain Res 44:73-85.

Ling TC, Mitrofanis J, Stone J (1989) The origin of retinal astrocytes in the rat: evidence of migration from the optic nerve. J Comp Neurol 286:345-352.

Linsenmeier RA, Yancey CM (1989) Effects of hyperoxia on the oxygen distribution in the intact cat retina. Invest Ophthalmol Visual Sci 30:612-618.
Michaelson IC (1954) Retinal circulation in man and animals. Springfield, IL: Thomas.

Millauer B, Wizigmann-Voos S, Schnürch H, Martinez R, Møller NHP Risau W, Ullrich A (1993) High affinity VEGF binding and developmental expression suggest Flk-1 as a major regulator of vasculogenesis and angiogenesis. Cell 72:835-846.

Millauer B, Shawver LK, Plate KH, Risau W, Ullrich A (1994) Glioblastoma growth inhibited in vivo by a dominant-negative Flk-1 mutant. Nature 576-579.

Minchenko A, Bauer T, Salceda S, Caro J (1994) Hypoxic stimulation of vascular endothelial growth factor expression in vitro and in vivo. Lab Invest 71:374.

Patel U (1983) Non-random distribution of blood vessels in the posterior region of the rat somatosensory cortex. Brain Res 289:75-70.

Peters KG, de Vries C, Williams LT (1993) Vascular endothelial growth factor receptor expression during embryogenesis and tissue repair suggests a role in endothelial differentiation and blood vessel growth. Proc Natl Acad Sci USA 90:8915-8919.

Phelps DL (1990) Oxygen and developmental retinal capillary remodeling in the kitten. Invest Ophthalmol Visual Sci 31:2194-2200.

Plate KH, Breier G, Widch HA, Risau W (1992) Vascular endothelial growth factor is a potential tumour angiogenesis factor in human gliomas in vivo. Nature 359:845-848.

Rapaport DH, Stone J (1984) The area centralis of mammalian retina: focal point for function and development of the visual system. Neuroscience 11:289-301.

Riddle DR, Gutierrez G, Zheng D, White LE, Richands A, Purves D (1993) Differential metabolic and electrical activity in the somatic sensory cortex of juvenile and adult rats. J Neurosci 13:4193-4213.

Schnitzer J (1988) Astrocytes in the guinea pig, horse, and monkey retina: their occurrence coincides with the presence of blood vessels. Glia 1:74-89.

Shweiki D, Itin A, Soffer D, Keshet E (1992) Vascular endothelial growth factor induced by hypoxia may mediate hypoxia-initiated angiogenesis. Nature 359:843-845.

Shweiki D, Itin A, Neufeld G, Gitay-Goren H, Keshet E (1993) Patterns of expression of vascular endothelial growth factor (VEGF) and VEGF receptors in mice suggest a role in homnonally regulated angiogenesis. J Clin Invest 91:2235-2243.

Simorre-Pinate V, Guerrin M, Chollet P, Penary M, Clamens S, Maleaze F, Plouet Jl (1994) Vasculotropin-VEGF stimulates retinal capillary endothelial cells through an autocrine pathway. Invest Ophthalmol Visual Sci.

Stone J, Dreher Z (1987) Relationship between astrocytes, ganglion cells and vasculature of the retina. J Comp Neurol 255:35-49.

Stone J, Itin A, Gnessin H, Chan-Ling T, Pe'er Y, Keshet E (1994) Normal retinal vascularisation is mediated by the hypoxia-induced expression of vascular endothelial growth factor (VEGF) by retinal glia. Proceedings of the XIth ISER Congress.

Terman BI, Carrion ME, Kovacs E, Rasmussen BA, Eddy RL, Shows TB (1991) Identification of a new endothelial cell growth factor receptor tyrosine kinase. Science 247:209-212.

Tout S, Chan-Ling T, Holländer H, Stone J (1993a) The role of Müller cells in the formation of the blood retinal barrier. Neuroscience 55: 291301.

Tout S, Dreher Z, Chan-Ling T, Stone J (1993b) Contact spacing among astrocytes is independent of neighbouring structures: in vivo and in vitro evidence. J Comp Neurol 332:433-443.

Watanabe T; Raff MC (1988) Retinal astrocytes are immigrants from the optic nerve. Nature 332:834-837.

Webster MJ, Rowe MH (1991) Disruption of developmental timing in the albino rat retina. J Comp Neurol 307:460-474.

Zheng D, LaMantia A-S, Purves D (1991) Specialized vascularization of the primate visual cortex. J Neurosci 11:2522-2529. 